Chirurg 2019 $\cdot 90: 418$

https://doi.org/10.1007/s00104-019-0961-x Online publiziert: 16. April 2019

(c) Springer Medizin Verlag GmbH, ein Teil von Springer Nature 2019

\section{Originalpublikation}

Noordmann BJ, Spaander MCW, Valkerna R et al (2018) Detection of residual disease after neoadjuvant chemoradiotherapy for esophageal cancer (preSANO): a prospective multicentre, diagnostic cohort study. Lancet Oncol 19:965-974

Hintergrund und Fragestellung. Nach neoadjuvanter Radiochemotherapie des Ösophaguskarzinoms ist nach der CROSS-Studie bei $49 \%$ der Plattenepithelkarzinome und $23 \%$ der Adenokarzinome kein Residualtumor in der histopathologischen Aufarbeitung mehr nachzuweisen. Diese Patienten mit histopathologischem "complete response“ (pCR) können mit den gegenwärtigen diagnostischen Methoden im Restaging nicht sicher identifiziert werden, sodass in den Leitlinien allen vorbehandelten Patienten weiterhin die transthorakale Ösophagektomie empfohlen wird. Ungeklärt bleibt gegenwärtig aber die Frage, ob Patienten mit pCR von einer onkologischen Resektion überhaupt profitieren. Von einer holländischen Arbeitsgruppe wurden jetzt die Ergebnisse einer prospektiven multizentrischen Kohortenstudien (preSANO-Trial) zum diagnostischen Nachweis eines pCR nach CROSS-Therapie vorgestellt.

Methoden. In dieser Untersuchung kamen insgesamt 207 Patienten mit Ösophaguskarzinom und neoadjuvanter Radiochemotherapie nach dem CROSSProtokoll zur Auswertung. Bei allen Patienten erfolgte 4 bis 6 Wochen nach CROSS ein Restaging (Ösophagogastroduodenoskopie mit regulären Biop-

W. Schröder · C. J. Bruns

Klinik für Allgemein-, Viszeral- und Tumorchirurgie, Universitätsklinik Köln, Köln, Deutschland

\title{
Nachweis von Residualtumor nach CROSS vor geplanter Ösophagektomie
}

sien [ÖGD], endoluminaler Ultraschall, [EUS]). Patienten mit nachgewiesenem Residualtumor und/oder Stenose wurden unmittelbar operiert. Bei den verbliebenen Patienten wurde ein zweites Restaging (ÖGD mit tiefer Biopsie, EUS, PositronenemissionstomographieComputertomographie [PET-CT], „fine needle aspiration" suspekter Lymphknoten [FNA]) und anschließend 10 bis 12 Wochen nach CROSS die Ösophagektomie durchgeführt. Primärer Endpunkt war die Korrelation des klinischen mit dem finalen histopathologischen Staging im Resektat (modifiziertes Tumorregressions-Grading nach Chirieac, TRG 3 und 4 definiert als $>10 \%$ Residualtumor).

Ergebnisse. Insgesamt $31 \%$ der Tumoren mit TRG 3 und 4 wurden mit der regulären Biopsie nach ÖGD und FNA nicht diagnostiziert. Bei dem Einsatz der ÖGD mit tiefen Biopsien (Bite-on-biteBiopsie) wurden lediglich $10 \%$ der TRG3- und -4-Tumoren (4 von 41 Patienten) im Restaging histologisch nicht gesichert. Der Einsatz des EUS mit Messung der maximalen Tumordicke konnte bei 11 von 39 Patienten (28\%) den Residualtumor nicht nachweisen. Das PET-CT konnte zwar bei 9\% der untersuchten Patienten distante Metastasen nachweisen, in Bezug auf den Residualturmor des Primärtumors wurden bei $15 \%$ der Patienten (6 von 41 TRG-3- und -4-Tumoren) falsch-negative Diagnosen gestellt. 123 Patienten dieser Serie hatten eine Endoskopie mit Bite-on-bite-Biopsie. In Kombination mit der FNA betrug für diese diagnostische Methode die Sensitivität und Spezifität 74 und $77 \%$, der positive Vorhersagewert für das Vorliegen eine
Residualtumors TRG 2-4 bzw. eine pCR wurde mit $92 \%$ berechnet (Konfidenzintervall 85-99\%).

Fazit. In dieser Studie wurden alle zur Verfügung stehenden diagnostischen Methoden eingesetzt, um nach neoadjuvanter Radiochemotherapie des Ösophaguskarzinoms einen Residualtumor nachzuweisen. Die Rate nichtdiagnostizierter Residualtumoren lässt sich durch die endoskopische Entnahme tiefer Biopsien gegenüber regulären Biopsien in Kombination mit der FNA deutlich reduzieren, sodass die Autoren diese endoskopische Technik für das Restaging nach CROSS-Therapie empfehlen. Die Studie bestätigt auch, dass das PET-CT zur Beurteilung des lokoregionären $\mathrm{Tu}$ mors nach neoadjuvanter Therapie nicht geeignet ist, aber seinen Stellenwert bei den Patienten hat, die einen Progress mit distanten Metastasen zeigen.

\section{Korrespondenzadresse}

Prof. Dr. W. Schröder, FACS, FEBS

Klinik für Allgemein-, Viszeral- und

Tumorchirurgie, Universitätsklinik Köln

Kerpener Str. 62, 50937 Köln, Deutschland

wolfgang.schroeder@uni-koeln.de

Interessenkonflikt. W.Schröder und C.J. Bruns geben an, dass kein Interessenkonflikt besteht. 